\title{
Susceptibility Calculations in Periodic and Quasiperiodic Planar Ising Models
}

\author{
Helen Au-Yang ${ }^{\mathrm{a}, 1}$ and Jacques H.H. Perk ${ }^{\mathrm{a}, 1}$ \\ ${ }^{a}$ Department of Physics, Oklahoma State University, 145 Physical Sciences, \\ Stillwater, Oklahoma 74078-3072, USA
}

\begin{abstract}
New results are presented for the wavevector-dependent susceptibility of $Z$-invariant periodic and quasiperiodic Ising Models in the scaling limit, generalizing old results of Tracy and McCoy for the square lattice. Explicit results are worked out for the two leading singular terms of the susceptibility on four regular isotropic lattices. The methods used provide a proof of the extended lattice-lattice scaling hypothesis for the class of models under consideration.
\end{abstract}

Key words: Ising model; $Z$-invariance; Wavevector-dependent susceptibility; Scaling limit; Painlevé functions; Corrections to scaling; Lattice-lattice scaling; Fibonacci sequences; Penrose tilings

\section{Introduction}

This paper is a brief report of results presented and discussed at a conference in Taiwan in honor of F.Y. Wu's seventieth birthday. Here we shall mainly present results that we have not published elsewhere. For the earlier results and a brief review of the literature we refer to our earlier papers [1-5].

The symmetric two-dimensional Ising model is defined by

$$
\mathcal{H}=-J \sum_{m, n}\left(\sigma_{m, n} \sigma_{m, n+1}+\sigma_{m, n} \sigma_{m+1, n}\right) .
$$

For this model one can define the elliptic moduli [6]

$$
k=\sinh ^{2}\left(2 J / k_{\mathrm{B}} T\right), \quad k^{\prime}=\sqrt{1-k^{2}},
$$

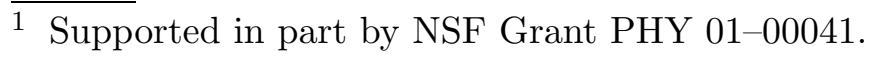


so that $k>1$ for $T<T_{\mathrm{c}}$ and $k<1$ for $T>T_{\mathrm{c}}$, while $k \rightarrow 1 / k$ gives the Kramers-Wannier duality transformation.

It is well-known that the spontaneous magnetization is given by $[7,8]$

$$
\langle\sigma\rangle=\left\{\begin{array}{cl}
\left(1-1 / k^{2}\right)^{1 / 8}, & T<T_{\mathrm{c}} \\
0, & T \geq T_{\mathrm{c}}
\end{array}\right.
$$

As pointed out by Baxter [9], this result generalizes in this form (3) to the general $Z$-invariant ferromagnetic Ising model, which includes the rectangular, triangular and honeycomb Ising lattices as special cases and the kagome lattice as a descendent [10-12]. The $Z$-invariant model is defined in terms of a set of oriented lines with line variables ("rapidities") $u_{1}, u_{2}, \cdots$, and spins on alternating faces. The nearest-neighbor "horizontal" and "vertical" couplings $K=\beta J$ and $\bar{K}=\beta \bar{J}$ are given by

$$
\begin{aligned}
& \sinh \left(2 K\left(u_{1}, u_{2}\right)\right)=k \operatorname{sc}\left(u_{1}-u_{2}, k^{\prime}\right)=\operatorname{cs}\left(\mathrm{K}\left(k^{\prime}\right)+u_{2}-u_{1}, k^{\prime}\right), \\
& \sinh \left(2 \bar{K}\left(u_{1}, u_{2}\right)\right)=\operatorname{cs}\left(u_{1}-u_{2}, k^{\prime}\right)=k \operatorname{sc}\left(\mathrm{K}\left(k^{\prime}\right)+u_{2}-u_{1}, k^{\prime}\right),
\end{aligned}
$$

where $\operatorname{sc}(v, k)=\operatorname{sn}(v, k) / \operatorname{cn}(v, k)=1 / \operatorname{cs}(v, k)$ are Jacobi elliptic functions and $\mathrm{K}(k)$ is the complete elliptic integral of the first kind [13]. For a further explanation of this model we refer to $[1,3,9,10]$.

\section{Correlations Functions in Baxter's $Z$-invariant Ising model}

The calculation of the pair correlation function $\left\langle\sigma_{m_{1}, n_{1}} \sigma_{m_{2}, n_{2}}\right\rangle$ can be carried out using quadratic difference equations [14]. In the $Z$-invariant model the correlation function is a function $g$ of the elliptic modulus and the rapidities of all oriented lines passing between the two spins. It satisfies

$$
\begin{aligned}
& k^{2} \operatorname{sc}\left(u_{2}-u_{1}, k^{\prime}\right) \operatorname{sc}\left(u_{4}-u_{3}, k^{\prime}\right) \\
& \quad \times\left\{g\left(u_{1}, u_{2}, u_{3}, u_{4}, \cdots\right) g(\cdots)-g\left(u_{1}, u_{2}, \cdots\right) g\left(u_{3}, u_{4}, \cdots\right)\right\} \\
& +\left\{g^{*}\left(u_{1}, u_{3}, \cdots\right) g^{*}\left(u_{2}, u_{4}, \cdots\right)-g^{*}\left(u_{1}, u_{4}, \cdots\right) g^{*}\left(u_{2}, u_{3}, \cdots\right)\right\}=0
\end{aligned}
$$

which is Eq. (7.2) of [4]. Here $g^{*}(m, n)$ is the dual correlation function obtained by replacing $k \rightarrow 1 / k$ and with spins on the dual lattice. The "..." stand for all other rapidity variables that are in common among all eight correlation functions in (6).

We are particularly interested in the scaling limit and its first correction. This 
means that we have to take the limit $k \rightarrow 1$ and ${k^{\prime}}^{2} \propto\left|T-T_{\mathrm{c}}\right| \rightarrow 0$. From [13] we find

$$
\frac{\pi}{2 \mathrm{~K}\left(k^{\prime}\right)}=k^{1 / 2}+\mathrm{O}\left(k^{\prime 4}\right), \quad q^{\prime}=\frac{1}{4} k^{2}+\mathrm{O}\left(k^{\prime 4}\right),
$$

so that

$$
k^{1 / 2} \operatorname{sc}\left(u, k^{\prime}\right)=\tan \left(\frac{\pi u}{2 \mathrm{~K}\left(k^{\prime}\right)}\right)+\mathrm{O}\left(k^{\prime 4}\right)=\tan (u)+\mathrm{O}\left(k^{\prime 2}\right) .
$$

Therefore, in order to take care of the leading corrections we need to amend the distance variable $R$ [1], originally conjectured by Bai-Qi Jin, so that the periodicity property of rapidity variables is reflected in it. Simply replacing each $u$ by $\pi u / 2 \mathrm{~K}\left(k^{\prime}\right)$, we now have

$$
R=\frac{1}{2}\left[\left\{\sum_{j=1}^{2 m} \cos \left(\pi u_{j} / \mathrm{K}\left(k^{\prime}\right)\right)\right\}^{2}+\left\{\sum_{j=1}^{2 m} \sin \left(\pi u_{j} / \mathrm{K}\left(k^{\prime}\right)\right)\right\}^{2}\right]^{1 / 2}
$$

We can then introduce the scaled distance $r$ using the diagonal correlation length $\xi_{\text {d }}$, i.e.,

$$
r=R / \xi_{\mathrm{d}}, \quad \text { where } \quad \xi_{\mathrm{d}}^{-1}=|\log k|
$$

The correlation functions take the limiting form

$$
g \equiv\left\langle\sigma \sigma^{\prime}\right\rangle \approx\left|1-k^{-2}\right|^{1 / 4} F(r), \quad g^{*} \equiv\left\langle\sigma \sigma^{\prime}\right\rangle^{*} \approx\left|1-k^{-2}\right|^{1 / 4} G(r),
$$

where the functions $F(r)$ and $G(r)$ satisfy

$$
F F^{\prime \prime}-F^{2}=-r^{-1} G G^{\prime}, \quad G G^{\prime \prime}-G^{2}=-r^{-1} F F^{\prime},
$$

and the front factor in (11) is the square of the spontaneous magnetization for $T<T_{\mathrm{c}}$ or $k>1$. $F(r)$ and $G(r)$ are the Painlevé functions for the uniform rectangular Ising lattice [20]. As discussed in [1,4], substituting (11) into (6) and expanding to leading nonvanishing order in $k^{\prime 2}$ shows that it is correct in the scaling limit $k=1$. For the leading correction we need to expand one order further leading to third-order differential equations. In order to show that these hold, there are some subtleties to take care of, that cannot be discussed in detail here. 


\section{Wavevector-Dependent Susceptibility in $Z$-Invariant Lattice}

For a general ferromagnetic $Z$-invariant lattice with $\mathcal{N}$ sites, the susceptibility $\chi$ is given by

$$
\bar{\chi} \equiv k_{\mathrm{B}} T \chi=\lim _{\mathcal{N} \rightarrow \infty} \frac{1}{\mathcal{N}} \sum_{m_{1}, n_{1}} \sum_{m_{2}, n_{2}}\left(\left\langle\sigma_{m_{1}, n_{1}} \sigma_{m_{2}, n_{2}}\right\rangle-\left\langle\sigma_{0,0}\right\rangle^{2}\right)
$$

where $\left(m_{1}, n_{1}\right)$ and $\left(m_{2}, n_{2}\right)$ run through the possible coordinates of the spins. In periodic cases one of the two sums can be done trivially. In quasiperiodic cases this can only be done asymptotically at the largest distance scale. Note that in the current model the spontaneous magnetization is site-independent and given by (3).

The wavevector-dependent susceptibility $\chi\left(q_{x}, q_{y}\right)$ is defined by

$$
\begin{aligned}
\bar{\chi}\left(q_{x}, q_{y}\right) & \equiv k_{\mathrm{B}} T \chi\left(q_{x}, q_{y}\right) \\
& =\lim _{\mathcal{N} \rightarrow \infty} \frac{1}{\mathcal{N}} \sum_{m_{1}, n_{1}} \sum_{m_{2}, n_{2}}\left(\left\langle\sigma_{m_{1}, n_{1}} \sigma_{m_{2}, n_{2}}\right\rangle-\left\langle\sigma_{0,0}\right\rangle^{2}\right) \mathrm{e}^{\mathrm{i}\left(q_{x} x+q_{y} y\right)},
\end{aligned}
$$

where $(x, y)$ is the physical distance vector between positions $\left(m_{1}, n_{1}\right)$ and $\left(m_{2}, n_{2}\right)$. Obviously, $\chi(0,0)$ is the usual susceptibility.

In the scaling limit, we can write

$$
\left\langle\sigma_{m_{1}, n_{1}} \sigma_{m_{2}, n_{2}}\right\rangle-\left\langle\sigma_{0,0}\right\rangle^{2}=\left|1-k^{-2}\right|^{1 / 4} F_{ \pm}(\kappa R)
$$

where

$$
\begin{aligned}
& F_{+}(\kappa R)=F\left(R / \xi_{\mathrm{d}}\right), \quad T>T_{\mathrm{c}}, \\
& F_{-}(\kappa R)=G\left(R / \xi_{\mathrm{d}}\right)-1, \quad T<T_{\mathrm{c}},
\end{aligned}
$$

and $\kappa=1 / \xi_{\mathrm{d}}=|\log k|$.

For periodic lattices we can choose suitable integer coordinates $M$ and $N$, to keep track of the repeating unit cell. For quasiperiodic lattices, this can only be done asymptotically. In addition, we need a multiplicity factor $g_{0}$ counting how many spin distance vectors fall exactly or asymptotically within a unit cell in the $(M, N)$ plane. Then

$$
\bar{\chi}\left(q_{x}, q_{y}\right) \approx g_{0}\left|1-k^{-2}\right|^{1 / 4} \int_{-\infty}^{\infty} \mathrm{d} M \int_{-\infty}^{\infty} \mathrm{d} N F_{ \pm}(\kappa R) \mathrm{e}^{\mathrm{i}\left(q_{x} x+q_{y} y\right)}
$$


with $(x, y)$ the physical distance vector corresponding to $(M, N)$.

In terms of the new coordinates $(M, N)$, we have

$$
R=\sqrt{a M^{2}+2 b M N+c N^{2}}
$$

with $a, b$, and $c$ known constants that can be calculated from (9). Using a linear transformation in the $(M, N)$ plane, we can arrive at $R^{2}=M^{\prime 2}+N^{\prime 2}$. Next, writing $M^{\prime}=R \cos \phi$ and $N^{\prime}=R \sin \phi$, we can replace the double integral over $M$ and $N$ by an integral over $R$ and an angle $\phi$, i.e.,

$$
\chi\left(q_{x}, q_{y}\right) \approx \frac{g_{0}\left|1-k^{-2}\right|^{1 / 4}}{\sqrt{a c-b^{2}}} \int_{0}^{2 \pi} \mathrm{d} \phi \int_{0}^{\infty} \mathrm{d} R R F_{ \pm}(\kappa R) \mathrm{e}^{\mathrm{i} Q R \cos \phi},
$$

where $Q=\sqrt{Q_{x}^{2}+Q_{y}^{2}}$ and $\left(Q_{x}, Q_{y}\right)$ follows by a linear transform from $\left(q_{x}, q_{y}\right)$. Rewriting this in the scaling variable $r=\kappa R=R / \xi_{\mathrm{d}}$, we get the final result

$$
\bar{\chi}\left(q_{x}, q_{y}\right) \approx \frac{g_{0}}{\sqrt{a c-b^{2}}} \frac{\left|1-k^{-2}\right|^{1 / 4}}{(\log k)^{2}} \int_{0}^{\infty} \mathrm{d} r r F_{ \pm}(r) \mathrm{J}_{0}(Q r / \kappa),
$$

with $\mathrm{J}_{0}(x)$ the Bessel function of index 0 . Eq. (21) generalizes a result of Tracy and McCoy [15-17] to (quasi)periodic $Z$-invariant lattices. It has three factors: The first factor contains the lattice anisotropy information. The second factor gives the divergence with the $-7 / 4$ exponent $[18,19]$. The last factor is the scaling function of Tracy and McCoy. Omitting the Bessel function in (21), as $J_{0}(0)=1$, gives the result for the ordinary susceptibility, generalizing the result of $\mathrm{Wu}$ et al. [20-23],

$$
\bar{\chi}=C_{0 \pm}\left|1-T_{\mathrm{c}} / T\right|^{-7 / 4}+C_{1 \pm}\left|1-T_{\mathrm{c}} / T\right|^{-3 / 4}+\mathrm{O}(1)
$$

with subscript + for $T>T_{\mathrm{c}}$ and - for $T<T_{\mathrm{c}}$, to all periodic and quasiperiodic $Z$-invariant Ising lattices.

This result also implies the universality of susceptibility amplitude ratios for all periodic and quasiperiodic ferromagnetic $Z$-invariant Ising models $[4,5]$. For the analysis of the long susceptibility series in the isotropic square lattice $C_{0+}$ and $C_{0-}$ were evaluated to very high precision by Nickel $[2,24]$. Therefore the corresponding accurate values for the isotropic triangular and honeycomb lattices can be obtained from the above formalism and they have already been reported by us [4,5], but as we shall work out the $C_{1 \pm}$ in the next section, we shall repeat the $C_{0 \pm}$ calculations in a slightly different way. Of course, results for various anisotropic $Z$-invariant lattices can be worked out also. 
For the isotropic square lattice we had $[2,4,5]$

$$
\begin{aligned}
& C_{0+}^{\mathrm{sq}}=0.9625817323087721140443298094334694951671391947579365 \\
& C_{0-}^{\mathrm{sq}}=0.02553697452202390538595345622639847192921968727077455 .
\end{aligned}
$$

To find the other numbers we only need to find their ratios with the squarelattice values,

$$
Q^{\mathrm{tr}} \equiv \frac{C_{0+}^{\mathrm{tr}}}{C_{0+}^{\mathrm{sq}}}=\frac{C_{0-}^{\mathrm{tr}}}{C_{0-}^{\mathrm{sq}}}=\frac{\lambda^{\mathrm{tr}}}{\lambda^{\mathrm{sq}}}, \quad Q^{\mathrm{hc}} \equiv \frac{C_{0+}^{\mathrm{hc}}}{C_{0+}^{\mathrm{sq}}}=\frac{C_{0-}^{\mathrm{hc}}}{C_{0-}^{\mathrm{sq}}}=\frac{\lambda^{\mathrm{hc}}}{\lambda^{\mathrm{sq}}},
$$

where - in view of (21) — for each lattice the corresponding $\lambda$ is given by

$$
\begin{aligned}
\lambda & =\frac{g_{0}}{\sqrt{a c-b^{2}}} \lim _{k \rightarrow 1} \frac{\left|1-k^{-2}\right|^{1 / 4}}{(\log k)^{2}}\left|1-T_{\mathrm{c}} / T\right|^{7 / 4} \\
& =\frac{4 g_{0}}{\sqrt{a c-b^{2}}} \lim _{k \rightarrow 1}\left|\frac{1-K / K_{\mathrm{c}}}{1-k^{2}}\right|^{7 / 4}=\left.\frac{4 g_{0}}{\sqrt{a c-b^{2}}}\left|K_{\mathrm{c}} \frac{\mathrm{d} k^{2}}{\mathrm{~d} K}\right|_{K=K_{\mathrm{c}}}\right|^{-7 / 4} .
\end{aligned}
$$

The $a, b, c$ and $g_{0}$ in (19) can be read off from [4]. For the square lattice, $a=c=1 / 2, b=0, g_{0}=1$, whereas for both the triangular and honeycomb lattices $a=c=3 / 4, b=-3 / 8$. Also, $g_{0}=1$ for the triangular lattice, but $g_{0}=2$ for the honeycomb lattice, as can be seen easily drawing both lattices as each other's dual. Hence,

$$
\begin{array}{ll}
k_{\mathrm{sq}}^{2}=\sinh ^{2}(2 K), & \lambda^{\mathrm{sq}}=\frac{2^{-11 / 8}}{\ln ^{7 / 4}(\sqrt{2}+1)}, \\
k_{\mathrm{tr}}^{2}=\sinh ^{3}(2 K)(2 \cosh (2 K)-\sinh (2 K)) \mathrm{e}^{4 K}, & \lambda^{\mathrm{tr}}=\frac{2^{3 / 2} 3^{-3 / 2}}{\ln ^{7 / 4} 3}, \\
k_{\mathrm{hc}}^{2}=\frac{\sinh ^{6}(2 K)}{(2 \cosh (2 K)-1)(\cosh (2 K)+1)^{2}}, & \lambda^{\mathrm{hc}}=\frac{2^{3 / 4} 3^{-5 / 8}}{\ln ^{7 / 4}(2+\sqrt{3})} .
\end{array}
$$

The above reproduces the numbers presented in [4,5]. Of course, these numbers agree within the reported accuracies with the series extrapolations of [26] and the four earlier values for the square and triangular lattices of $[15,20-23]$. They also agree to better than three places with those obtained from the Syozi-Naya 
approximation [25] for $T$ above $T_{\mathrm{c}}$, which is precisely the $\chi_{<}^{(1)}$ approximation in $[20]$.

During the conference, Prof. Guttmann asked us to check if these numbers agree with the corresponding results [27-34] using the lattice-lattice scaling hypothesis. They do agree and the current work can be seen as a theoretical confirmation of the heuristic lattice-lattice scaling approach to susceptibility amplitude calculations in planar Ising models. In the next section we shall see that the results for the kagome lattice and for the $C_{1 \pm}$ also agree.

\section{Susceptibility Amplitudes and Lattice-Lattice Scaling}

Lattice-lattice scaling - or the generalized law of corresponding states - was first introduced by Betts, Guttmann and Joyce [27]. Guttmann extended the method to predict the $C_{0 \pm}$ and $C_{1 \pm}$ for the triangular and honeycomb lattices $[28,30]$. Ritchie and Betts showed that the method fails for the kagome lattice [31], but this was fixed by Guttmann who introduced the extended latticelattice scaling hypothesis [33].

The reason why the kagome lattice is different can be understood from the fact that the kagome Ising model is not a $Z$-invariant model as such, but that it is a descendent of the $Z$-invariant honeycomb lattice model after decoration and star-triangle transformation [10-12]. This results in

$$
\chi^{\mathrm{ka}}(K)=\frac{3}{2}\left(1-\frac{4}{\left(\mathrm{e}^{4 K}+1\right)^{2}}\right) \chi^{\mathrm{hc}}\left(K^{\mathrm{hc}}\right)+\mathrm{O}(1)
$$

where

$$
K^{\mathrm{hc}}=\frac{1}{2} \log \frac{\mathrm{e}^{4 K}+1}{2} .
$$

There is a front factor to the $\chi^{\mathrm{hc}}\left(K^{\mathrm{hc}}\right)$ in $(27)$ that contributes nontrivially to the $C_{1 \pm}^{\mathrm{ka}}$ and is inconsistent with simple lattice-lattice scaling.

For the kagome lattice, it is well known that $K_{\mathrm{c}}^{\mathrm{ka}}=\frac{1}{4} \ln (3+2 \sqrt{3})$ gives its critical point. Therefore, the critical value of the front factor in $(27)$ is $6 \sqrt{3}-9$. The remainder of the calculation of the $C_{0 \pm}^{\mathrm{ka}}$ uses (25) with (28) substituted in the elliptic modulus (26) of the honeycomb lattice. This leads to the ratios

$$
Q^{\mathrm{ka}} \equiv \frac{C_{0+}^{\mathrm{ka}}}{C_{0+}^{\mathrm{sq}}}=\frac{C_{0-}^{\mathrm{ka}}}{C_{0-}^{\mathrm{sq}}}=\frac{\lambda^{\mathrm{tr}}}{\lambda^{\mathrm{sq}}}, \quad \text { with } \quad \lambda^{\mathrm{ka}}=\frac{2^{5 / 2}(2-\sqrt{3})}{\ln ^{7 / 4}(3+2 \sqrt{3})} .
$$


To calculate the various $C_{1 \pm}$, we note that we can obtain the ratios $C_{1 \pm} / C_{0 \pm}$ from the middle factor in $(21)$. For $\left(q_{x}, q_{y}\right)=(0,0)$, the last factor is constant, and the first factor does not contribute in first order since we improved the $R$ variable as in (9), so that also the $a, b$ and $c$ in (19) are constants in this order. Using the new variable $z=\exp (2 K)$, we can reexpress the elliptic moduli $k$ and calculate the critical values $z_{\mathrm{c}}$. Expanding the middle factor in $(21)$ and the front factor of (27) to leading and next-leading order, we find

$$
\begin{array}{lll}
k^{\mathrm{sq}}=\frac{\left(z^{2}-1\right)^{2}}{4 z^{2}}, & z_{\mathrm{c}}^{\mathrm{sq}}=\sqrt{2}+1, & R^{\mathrm{sq}}=\frac{\sqrt{2} \ln (\sqrt{2}+1)}{16}, \\
k^{\mathrm{tr}}=\frac{\left(z^{2}-1\right)^{3 / 2}\left(z^{2}+3\right)^{1 / 2}}{4 z}, & z_{\mathrm{c}}^{\mathrm{tr}}=\sqrt{3}, & R^{\mathrm{tr}}=\frac{\ln 3}{16}, \\
k^{\mathrm{hc}}=\frac{(z-1)^{3}(z+1)}{4\left(z^{2}-z+1\right)^{1 / 2} z^{3 / 2}}, & z_{\mathrm{c}}^{\mathrm{hc}}=2+\sqrt{2}, & R^{\mathrm{hc}}=\frac{\sqrt{3} \ln (2+\sqrt{3})}{24}, \\
k^{\mathrm{ka}}=\frac{\left(z^{2}-1\right)^{3}\left(z^{2}+3\right)}{2^{7 / 2}\left(z^{4}+3\right)^{1 / 2}\left(z^{2}+1\right)^{3 / 2}}, & z_{\mathrm{c}}^{\mathrm{ka}}=\sqrt{3+2 \sqrt{2}}, & R^{\mathrm{ka}}=\frac{(\sqrt{3}-1) \ln (3+2 \sqrt{3})}{16} .
\end{array}
$$

Here, for each lattice, the parameter $R^{\mathrm{xx}}$ determines the ratios

$$
\frac{C_{1+}^{\mathrm{xx}}}{C_{0+}^{\mathrm{xx}}}=-\frac{C_{1-}^{\mathrm{xx}}}{C_{0-}^{\mathrm{xx}}}=R^{\mathrm{xx}}, \quad \text { with } \mathrm{xx}=\mathrm{sq}, \text { tr, he, ka. }
$$

Again, these results agree with Guttmann's extended lattice-lattice scaling results $[28,31,33]$ and provide a deeper understanding why this is so.

\section{Additional Remarks}

The current project was started because of renewed interest in the calculation of susceptibilities in planar Ising models. First, we have done computations on quasiperiodic lattices. For the so-called Fibonacci Ising models [1,3], the lattice is still periodic, whereas the coupling constants vary according to Fibonacci sequences. For the purely ferromagnetic case we found not much structure in the wavevector-dependent susceptibility, whereas in the mixed-sign cases more and more incommensurate peaks show up as one approaches the critical point. More recently, we also have results for the Ising model with spins on alternating sites of a Penrose tiling, which we shall publish elsewhere. Here, the wavevector-dependent susceptibility shows a pattern with several peaks and a ten-fold symmetry even in the ferromagnetic case. Therefore, if the underlying lattice is periodic, there may be incommensurate structure within a unit cell in $\left(q_{x}, q_{y}\right)$ space. But, if the underlying lattice is aperiodic, so will the pattern be in $\left(q_{x}, q_{y}\right)$ space. However, the above result (21) applies to all these quasiperiodic $Z$-invariant cases.

The second reason for coming back to the Ising model was to examine an 
intriguing result of Aharony and Fisher [35]. Their work shows no indication for the presence of irrelevant variables in the isotropic Ising models, which - if correct to all orders - leads to a prediction of all power-law singular terms in the susceptibility. Having more powerful computers at our disposal the authors of [2] used algorithms of polynomial complexity based on [36] to generate highand low-temperature series to order 323 and several terms in the asymptotic expansions near $T_{\mathrm{c}}$. It should be noted that only the last few digits of each next coefficient has new information, so that we had to work with very high precision arithmetic. Due to our special algorithms we could do the numerical evaluations in polynomial time. In this sense, polynomial algorithms are just as good as having closed-form formulae.

We now know that this simple picture of no irrelevant variables is incomplete and that corrections to scaling due to breaking of rotational symmetry must be considered. Indeed, the correlation functions have a kind of multipole longdistance expansion [36], which can explain the deviations from fourth order on. Very recently, a conformal field theory explanation has also been given [37]. To study the effects of the irrelevant variables in more detail we shall have to study the Ising model on lattices other than the isotropic square lattice.

Another interesting feature discussed in more detail in [2] is that the critical temperature is part of a natural boundary for the susceptibility. This means that there exists a closed curve of essential singularities prohibiting analytic continuation in the complex temperature plane from high to low temperatures. It can, therefore, be understood why there is no simple closed form expression available after more than half a century of research. But, there is now no need for such expressions having algorithms of polynomial complexity available.

\section{Acknowledgements}

The authors thank Prof. A.J. Guttmann for useful suggestions.

\section{References}

[1] H. Au-Yang, B.-Q. Jin and J.H.H. Perk, J. Stat. Phys. 102 (2001) 501-543.

[2] W.P. Orrick, B.G. Nickel, A.J. Guttmann and J.H.H. Perk, J. Stat. Phys. 102 (2001) 795-841; Phys. Rev. Lett. 86 (2001) 4120-4123. ${ }^{2}$

$\overline{2}$ For the additional series coefficients, see http://www.ms.unimelb.edu.au/ ${ }^{\sim}$ tonyg . 
[3] H. Au-Yang and J.H.H. Perk, in MathPhys Odyssee 2001: Integrable Models and Beyond, T. Miwa and M. Kashiwara, eds., (Birkhäuser, Boston, 2002), pp. $1-21$.

[4] H. Au-Yang and J.H.H. Perk, in MathPhys Odyssee 2001: Integrable Models and Beyond, T. Miwa and M. Kashiwara, eds., (Birkhäuser, Boston, 2002), pp. $23-48$.

[5] H. Au-Yang and J.H.H. Perk, Intern. J. Mod. Phys. B 16 (2002) 2089-2095.

[6] L. Onsager, Phys. Rev. 65 (1944) 117-149.

[7] L. Onsager, Nuovo Cimento (ser. 9) 6 (Suppl.) (1949) 261; C.N. Yang, Phys. Rev. 85 (1952) 808-816.

[8] B.M. McCoy and T.T. Wu, The Two-Dimensional Ising Model, (Harvard Univ. Press, Cambridge, Mass., 1973).

[9] R.J. Baxter, Phil. Trans. R. Soc. Lond. A 289 (1978) 315-346.

[10] H. Au-Yang and J.H.H. Perk, Physica A 144 (1987) 44-104.

[11] M.E. Fisher, Phys. Rev. 113 (1959) 969-981.

[12] I. Syozi and S. Naya, Progr. Theor. Phys. 24 (1960) 829-839.

[13] I.S. Gradshteyn, I.M. Ryzhik and A. Jeffrey, Table of Integrals, Series, and Products, Fifth Edition, (Academic Press, London, 1996), see particularly Eqs. 8.113.1, 8.146.8 and 8.197.3.

[14] J.H.H. Perk, Phys. Lett. 79A (1980) 3-5.

[15] C.A. Tracy and B.M. McCoy, Phys. Rev. Lett. 31 (1973) 1500-1504.

[16] C.A. Tracy and B.M. McCoy, Phys. Rev. B 12 (1975) 358-387.

[17] C.A. Tracy, AIP Conference Proceedings 29 (1975) 483-487.

[18] M.E. Fisher, Physica 25 (1959) 521-524.

[19] M.E. Fisher and R.J. Burford, Phys. Rev. 156 (1967) 583-622.

[20] T.T. Wu, B.M. McCoy, C.A. Tracy and E. Barouch, Phys. Rev. B 13 (1976) $316-374$.

[21] E. Barouch, B.M. McCoy and T.T. Wu, Phys. Rev. Lett. 31 (1973) 1409-1411.

[22] B.M. McCoy, C.A. Tracy and T.T. Wu, J. Math. Phys. 18 (1977) 1058-1092.

[23] H.G. Vaidya, Phys. Lett. A 57 (1976) 1-4.

[24] B. Nickel, J. Phys. A 32 (1999) 3889-3906; A 33 (2000) 1693-1711.

[25] I. Syozi and S. Naya, Progr. Theor. Phys. 24 (1960) 374-376.

[26] M.F. Sykes, D.G. Gaunt, P.D. Roberts and J.A. Wyles, J. Phys A 5 (1972) 624-639. 
[27] D.D. Betts, A.J. Guttmann and G.S. Joyce, J. Phys. C 4 (1971) 1994-2008.

[28] A.J. Guttmann, Phys. Rev. B 9 (1974) 4991-4492; B 12 (1975) 1991.

[29] C.J. Thompson and A.J. Guttmann, Phys. Lett. 53A (1975) 315-316.

[30] D.S. Ritchie and D.D. Betts, Phys. Rev. B 11 (1975) 2559-2563.

[31] A.J. Guttmann, J. Phys. A 8 (1975) 1236-1248.

[32] A.J. Guttmann, J. Phys. A 8 (1975) 1249-1255.

[33] A.J. Guttmann, J. Phys. A 10 (1977) 1911-1916.

[34] D.S. Gaunt and A.J. Guttmann, J. Phys. A 11 (1978) 1381-1397.

[35] A. Aharony and M.E. Fisher, Phys. Rev. Lett. 45 (1980) 679-682; Phys. Rev. B 27 (1983) 4394-4400.

[36] H. Au-Yang and J.H.H. Perk, Phys. Lett. A 104 (1984) 131-134; X.-P. Kong, Ph.D. thesis, Stony Brook (1987).

[37] M. Caselle, M. Hasenbusch, A. Pelissetto and E. Vicari, J. Phys. A 35 (2002) $4861-4888$. 